\title{
Klaus Meier Positionierung der katholischen Kirche im Internet
}

Das Internet hat sich zu einem Massenmedium entwickelt, das in allen gesellschaftlichen Milieus zu Hause ist - allerdings mit ganz unterschiedlicher Dichte. Während zum Beispiel nur 15 Prozent der Konservativen und fünf Prozent der Traditionsverwurzelten das Internet nutzen sind es im Milieu der Modernen Performer 77 Prozent und bei den Postmateriellen und den Experimentalisten rund zwei Drittel. ${ }^{1}$ Eine InternetStrategie der katholischen Kirche sollte sich also auf die Einstellungen, Wünsche und Erwartungen ganz bestimmter Milieus konzentrieren. Traditionelle Lebensauffassungen und Lebensstile - kurzum: der kirchliche Mainstream - spielen beim Internet-Publikum eine sehr geringe Rolle. Die Internet-Nutzer wünschen sich einerseits eine offene Kirche („Kirche von unten“) und sehen andererseits Kirche als „virtuelle Basisstation“, mit der sie sich neben anderen Religionen, Weltanschauungen und Philosophien autonom und kreativ auseinandersetzen und die sie in Krisenzeiten beanspruchen möchten.

Für eine differenzierte Analyse möglicher Bausteine einer InternetStrategie der katholischen Kirche werden im Folgenden zunächst Nutzung, Spezifika und Trends des neuen Mediums Internet umrissen. Danach wird die Positionierung der katholischen Kirche in den Sinus-Milieus zusammengefasst, in denen das Internet am intensivsten genutzt wird. Auf Basis dieser beiden Säulen werden schließlich am Ende Empfehlungen erarbeitet.

\section{Das Internet: Nutzung und Spezifika eines neuen Mediums}

58 Prozent der Deutschen ab 14 Jahren (37 Millionen Menschen) nutzen inzwischen zumindest gelegentlich das Internet ${ }^{2}$, wöchentlich sind es

1 Grundlage dieser Analyse ist die Studie zu „Religiösen und kirchlichen Orientierungen in den Sinus-Milieus", die von der Sinus Sociovision in Heidelberg in Auftrag der Medien-Dienstleistungs GmbH der katholischen Kirche im Jahr 2005 durchgeführt wurde (vgl. MDG/Sinus Sociovision: Milieuhandbuch. Religiöse und kirchliche Orientierungen in den Sinus-Milieus. München, Heidelberg 2005). Im Folgenden als "Milieuhandbuch" bezeichnet.

2 Vgl. Birgit van Eimeren/Beate Frees: Nach dem Boom: Größter Zuwachs in internetfernen Gruppen. ARD/ZDF-Online-Studie 2005. In: Media Perspektiven, Jg. 2005 , H. 8, S. 362-379, hier S. 363. 
knapp 47 Prozent, täglich etwa 30 Prozent. $^{3}$ Am intensivsten ist das Medium bei Jugendlichen und jungen Erwachsenen verbreitet: 96 Prozent der 14- bis 19-Jährigen und 85 Prozent der 20-•bis 29-Jährigen bezeichnen sich als Internet-Nutzer. Das Internet liegt in diesen Altersgruppen weit vor der Reichweite der Tageszeitungen (49 bzw. 60 Prozent). ${ }^{4}$ Aber selbst im Querschnitt der Gesamtbevölkerung hat das Internet in der Nutzungsdauer die Zeitung überholt: Nach der repräsentativen Studie „Massenkommunikation 2005“ lesen die Deutschen im Schnitt täglich 28 Minuten lang die Tageszeitung und sind 44 Minuten lang online. Die „Veralltäglichung des Internetgebrauchs" schreitet demnach immer weiter voran. ${ }^{5}$ Die Bindung an das Internet - gemessen über die so genannte „Inselfrage“ - ist mittlerweile höher als an die Tageszeitung: 16 Prozent würden sich für das Internet entscheiden, wenn sie nur noch ein Medium behalten könnten, nur zwölf Prozent für die Tageszeitung; weiter führend sind Fernsehen (44 Prozent) und Radio (26 Prozent), wobei die Bindung an das Fernsehen mit der Zeit abgenommen hat. ${ }^{6}$ Menschen aller Altergruppen nutzen das Internet überwiegend, weil sie sich informieren möchten; vor allem bei Jüngeren spielt darüber hinaus der Spaßfaktor eine bedeutende Rolle - hier liegt das Internet bei den 14- bis 29-Jährigen fast gleichauf mit dem Fernsehen. ${ }^{7}$

Welche Erlebnisweisen bietet das Internet? Nach einer Studie von ZDF und forsa erweist sich für das Internet die Orientierungsleistung als die wichtigste Dimension: ${ }^{8}$ Das Internet gibt vor allem jungen Menschen nicht nur „neue Informationen“, sondern auch „Anregungen und Stoff zum Nachdenken“. Das soziale Erleben ist mit dem Internet deutlich stärker ausgeprägt als mit dem Fernsehen: Das Internet hilft nach Aussagen der Nutzer, „sich im Alltag zurechtzufinden“, und gibt ihnen „das

3 Vgl. Arbeitsgemeinschaft Online Forschung (AGOF): Berichtsband - Zusammenfassung zur internet facts 2005-III, S. 5.

4 Vgl. Anja Pasquay: Zur Lage der Zeitungen in Deutschland 2005 (http://www. bdzv.de/markttrends_und_daten.html; 22.5.2006).

5 Vgl. Christa-Maria Ridder/Bernhard Engel: Massenkommunikation 2005: Images und Funktionen der Massenmedien im Vergleich. Ergebnisse der 9. Welle der ARD/ZDF-Langzeitstudie zur Mediennutzung und -bewertung. In: Media Perspektiven, Jg. 2005, H. 9, S. 422-448, hier S. 425 und 429.

6 Vgl. Birgit van Eimeren/Christa-Maria Ridder: Trends in der Nutzung und Bewertung der Medien 1970 bis 2005. Ergebnisse der ARD/ZDF-Langzeitstudie Massenkommunikation. In: Media Perspektiven, Jg. 2005, H. 10, S. 490-504, hier S. 493.

7 Vgl. Ridder/Engel: Massenkommunikation 2005, a. a. 0., S. 428-430.

8 Vgl. Ursula Dehm/Dieter Storll/Sigrid Beeske: Das Internet: Erlebnisweisen und Erlebnistypen. Sich ergänzende und konkurrierende Gratifikationen durch Fernsehen und Internet. In: Media Perspektiven, Jg. 2006, H. 2, S. 91-101. 
Gefühl dazuzugehören“. Darüber hinaus sind für einen Teil der InternetNutzer Personen, denen sie in diesem Medium begegnen, „wie gute Freunde für mich". Gerade in dieser Suche nach Orientierung und sozialem Erleben im Internet können Religionsgemeinschaften und Kirchen Anknüpfungspunkte finden.

Das neue Medium Internet ist ein Spiegel der Multioptionsgesellschaft. Jeder Mensch, jede Organisation, jedes Unternehmen kann sich im Internet präsentieren, Marketing betreiben oder sich an Diskussionen beteiligen. Es bietet auch der katholischen Kirche die Möglichkeit, an den traditionellen Schleusenwärtern der Massenkommunikation - den Journalisten - vorbei mit Gläubigen und Ungläubigen, mit Kritikern, Zweiflem und Enthusiasten ins Gespräch zu kommen. In Deutschland präsentieren sich katholische Gemeinden, Diözesen, kirchliche Einrichtungen, Verbände oder Laien-Netzwerke auf zumindest hunderten, wenn nicht sogar tausenden Websites. Der nie gekannten Angebotsfülle im Internet steht allerdings ein Kampf um Aufmerksamkeit gegenüber. ${ }^{9}$ Auch für nicht-journalistische Anbieter ist „die Lenkung der Aufmerksamkeit auf die eigene Website ein entscheidender Erfolgsfaktor"10.

Das Internet ist permanent in Bewegung. Folgende drei Trends, die zurzeit die Diskussion um die soziale und technische Entwicklung des Internets mit bestimmen, sind für unser Thema relevant:

- Interaktivität und Partizipation: Das Internet ist das erste Medium, das dem Brechtschen Ideal einer dialogischen, gleichberechtigten Kommunikation zwischen Sender und Empfänger - zumindest technisch - nahe kommt. Nach einem Jahrzehnt eher enttäuschender Erfahrungen mit diesem Potenzial bieten nun neue Formate für eine breite Masse der Internet-Nutzer die Möglichkeit, einfach und sehr kostengünstig oder kostenfrei selbst zu publizieren, sich mit anderen Inhalten $\mathrm{zu}$ vernetzen und $\mathrm{zu}$ diskutieren. ${ }^{11}$ Unter den Begriffen Social Software oder Web 2.0 werden üblicherweise Anwendungen zusammengefasst wie Weblogs, Podcasts, Wikis, Social Bookmarks oder Community-Plattformen. Weblogs zum Beispiel sind eine Mischung aus Tagebuch-Einträgen, Kommentaren und Netzfunden.

9 Vgl. Klaus Beck/Wolfgang Schweiger (Hg.): Attention please! Online-Kommunikation und Aufmerksamkeit. München 2001.

10 Christoph Neuberger/Thomas Pleil: Online-Public Relations: Forschungsbilanz nach einem Jahrzehnt. In: Klaus Merten/ Elke Neujahr (Hg.): Handbuch der Unternehmenskommunikation 2005. Münster 2006 (in Druck).

11 Vgl. 2.B. Thomas Pleil/Ansgar Zerfaß: Internet und Social Software in der Unternehmenskommunikation. In: Manfred Piwinger/Ansgar Zerfaß (Hg.): Handbuch Unternehmenskommunikation. Wiesbaden 2006 (in Druck). 
Podcasts sind Audio-Beiträge, die Nutzer auf dem Computer oder auf tragbaren MP3-Playern - wie dem iPod von Apple oder auf immer mehr Mobiltelefonen - speichern können. Weblogs und Podcasts können untereinander verlinkt - und meist von den Nutzern kommentiert werden. Sehr oft findet sich in Weblogs ein buntes Sammelsurium an Selbstdarstellung - mitunter aber intensive, informative und emotionale Glaubensdokumentation. Der. OnlineJournalismus, vor allem in den USA, greift die neuen interaktiven Formen auf und entwickelt zurzeit Formen des Citizen Journalism, die Nutzern vielfältige Beteiligung an journalistischen Angeboten ermöglichen. ${ }^{12}$ Neuestes Beispiel in Deutschland ist die „Readers Edition" der netzeitung.de, die im Juni 2006 gestartet ist (www. readers-edition.de). In den USA setzen auch Religionsgemeinschaften zunehmend diese Beteiligungsformen im Internet ein.

- Video: Gut ein Drittel der Internet-Nutzer in Deutschland verfügt mittlerweile über einen Breitband-Zugang ${ }^{13}$, der technisch gute Video-Qualität ermöglicht. Ebenfalls etwa ein Drittel bezahlt den Internetanschluss zeitunabhängig (Flatrate) - der Blick auf die Uhr entfällt. Das Interesse an Videos im Netz ist in den vergangenen Monaten weltweit enorm gestiegen. Das beherrschende Thema einer Tagung der Europäischen Nachrichtenagenturen im Mai 2006 in Wien war die wachsende Nachfrage nach Videos von OnlineAnbietern jeglicher Couleur. ${ }^{14}$ "Spiegel online“ zum Beispiel nutzt den Trend und bietet inzwischen Videos, die direkt in die Startseite integriert sind. Chefredakteur Mathias Müller von Blumencron: "Videos sind das emotionalste, was man im Netz machen kann."15 Video im Internet heißt aber nicht, das traditionelle Fernsehen 1:1 zu übertragen, sondern neue Formen des Multimedia-Storytellings $z u$ entwickeln, die Text, Ton, animierte Grafik und Video kombinieren. ${ }^{16}$

12 Vgl. Steve Outing: The 11 Layers of Citizen Journalism. (http://www.poynter.org/ content/content_view.asp?id=83126; 22.5.2006).

13 Vgl. van Eimeren/Frees: Nach dem Boom, a. a. 0., S. 371.

14 Die Nachrichtenagenturen produzieren traditionell nur Text, Bild, Grafik und vereinzelt Radiodienste. Einzig Reuters stellt schon seit Jahrzehnten Video-Feeds zur Verfügung. Seit kurzem liefern neben den Weltagenturen AFP und AP z.B. auch die nationalen Nachrichtenagenturen TT in Schweden, ANSA in Italien, PA in Großbritannien oder CTK in Tschechien Videoservices für Online-Anbieter. Die Videos können z.T. in Online-Shops gekauft und auf der eigenen Website verwendet werden.

15 Mathias Müller von Blumencron: „Wir sind positiv nervös“. In: Journalist, 56. Jg. 2006, H. 5, S. 52-55, hier S. 54.

16 Vgl. Klaus Meier (Hg.): Internet-Journalismus. 3. Aufl. Konstanz 2002, S. 129-151. 
- Service und Transaktion: Neben den medialen und kommunikativen Funktionen ist das Internet bei - traditionell nicht-medialen Transaktionen fest im Alltag verankert. Homebanking, Onlineauktionen (z.B. auf der Versteigerungsplattform Ebay), Onlineshopping, Download von Formularen und Anträgen, Musik und Video gehört bei vielen Internet-Nutzern zum Alltag. ${ }^{17}$ Ganze Geschäftsprozesse von Unternehmen und Organisationen haben sich auf Internet und Intranet verlagert.

\section{Positionierung der katholischen Kirche in den Internet- Milieus}

Mehrere aktuelle Studien bestätigen, was im Milieuhandbuch am Rande thematisiert wird: ${ }^{18}$ Das Internet ist zurzeit vor allem das Medium der Mittel- und Oberschicht sowie der Menschen, die in der Werteachse der Grundorientierung $C$ und in Teilen B zugeordnet werden können. In der zweidimensionalen Verortung der Sinus-Milieus liegt der ganz große Teil der Internet-Nutzer demnach rechts oben - in Richtung links unten nehmen sie immer mehr ab. Nach der Studie e-milieu sind mehr als die Hälfte der Internet-Nutzer Moderne Performer oder Postmaterielle; knapp 20 Prozent sind Experimentalisten. Die restlichen 30 Prozent verteilen sich auf die anderen Milieus - wobei davon der Großteil in der Bürgerlichen Mitte und bei den Etablierten zu suchen ist (jeweils ca. neun Prozent). In den konservativen Milieus (Traditionsverwurzelte, Konservative, DDRNostalgische) sowie in den auf Modernisierung und Neuorientierung eingestellten Milieus der Unterschicht (Konsum-Materialisten und Hedonisten) findet sich generell eine sehr geringe Internet-Nutzung.

Das Milieuhandbuch gliedert die Positionierung und Bedeutung der katholischen Kirche in vier Bereiche (S.15). Beim Vergleich mit der Internet-Nutzung zeigt sich sehr schnell, dass das Internet sowohl im kirchlichen Mainstream als auch in kirchenfernen Milieus weniger genutzt wird als in Milieus, die ein partizipatives und "virtuelles“ Interesse an der Kirche haben:

17 Vgl. van Eimeren/Frees: Nach dem Boom, a. a. 0., S. 371; AGOF: Berichtsband, a. a. $0 .$, S. 11.

18 Vgl. Sinus Sociovision/Media Transfer: e-milieu (http://www.sinus-sociovision.de/ 2/2-3-3-11.htm; 24.5.2006); Bernhard Engel/Thomas Windgasse: Mediennutzung und Lebenswelten 2005. Ergebnisse der 9. Welle der ARD/ZDF-Langzeitstudie „Massenkommunikation“. In: Media Perspektiven, Jg. 2005, H. 9, S. 449-464. 
- Kirchlicher Mainstream: Die Kirche ist hier Zentrum, Verwalter und Vermittler des Glaubens mit exklusiver Geltung. Mit dieser Botschaft der „Volkskirche“ können allerdings nur wenige "Internet-Nutzer erreicht werden. Wenn überhaupt, dann die Etablierten, welche die Kirche als Hüter kultureller Errungenschaften bewundern und gerne kirchliche Museen und Kathedralen besuchen.

- Kirchendistanz: Die kruden und provokanten Kritiker der Kirche im Milieu der Hedonisten sowie die Konsum-Materialisten, die ihr Grundwissen zum Glauben - sofern überhaupt vorhanden - aus Spielfilmen beziehen, können ebenfalls mit dem Internet kaum erreicht werden. Die Images und Wünsche von der Kirche als sozial-karikativer Rettungsanker oder als Hilfe für existentielle Lösungen, die hier vorherrschen, müssen demnach nicht unbedingt über das Internet kommuniziert werden.

- Utopie von Kirche: Im diesem großen mittleren Segment ist die Auseinandersetzung mit der Kirche sehr engagiert - emotional und rational. Es besteht ein „ausgeprägtes Interesse an der Umgestaltung der Kirche in Richtung mehr persönlicher Partizipation, Gestaltungsfreiheit und Verantwortung" (S. 16). Der nachdrückliche Wunsch nach einer umfassenden Umgestaltung von Kirche, „damit sie so werden kann, wie sie von Gott gewollt ist und für die Menschen gut ist" (S. 17), trifft auf etwa ein Drittel der Internet-Nutzer zu - vor allem auf Vertreter des postmateriellen Milieus („Kirche auf dem Weg“). Die Internet-Kommunikation sollte also zu einem Teil genau dieses Milieu ansprechen. Das Engagement für Fragen nach dem Sinn des Lebens ist in diesem Milieu am stärksten ausgeprägt. Dort wird die Kirche als ein Projekt von Menschen auf der Suche nach dem richtigen Leben gesehen. Man wünscht sich eine Intensivierung des interreligiösen Dialogs, Ökumene, Aufhebung des Zölibats, Priesteramt für Frauen und demokratische Strukturen (Milieuhandbuch, S. 18). Glaubwürdige Kirchenvertreter könnten hier über das Internet kommunizieren, dass sie zwar von der Utopie eines guten Lebens überzeugt sind, dass sie aber weiter an sich arbeiten und jeder auf dem Weg zur „endgültigen Wahrheit" ist (S. 61). Eine Kommunikationsfalle wäre $\mathrm{zu}$ signalisieren, dass man endgültig „angekommen“ ist und die eigene Perspektive die allein richtige ist. Kirchliche Themen könnten dagegen in Verbindung mit politischen Utopien von sozialer Gerechtigkeit und Ökologie kommuniziert werden, z.B. die Forderungen nach einem weltweiten Ende von Armut, Hunger, Kinderarbeit und Wirtschaftsimperialismus. Kirchliche Websites könnten, um dieses Milieu im positiven Sinne zu erreichen, generell gesellschaftspolitische Themen aufgreifen (S. 81). 
Auch Themen wie ganzheitliche Gesundheit, neue Jugend- und Medienkulturen bewegen dieses Milieu.

- Kirche als virtuelles Instrument: In diesem Segment ist die Herausforderung für eine gelingende Internet-Kommunikation am größten. Denn einerseits befindet sich hier etwa die Hälfte aller InternetNutzer (vor allem die sehr jungen Modernen Performer), andererseits lässt sich hier ein sehr unengagiertes, autonomes, individuelles und instrumentelles Verhältnis zur Kirche feststellen (S. 19): Man befolgt nicht die Regeln der Kirche, sondern macht Kirche zu einem variablen Parameter des eigenen Regelwerks. Kirche ist ein Dienstleister - eine Art „virtuelle Basisstation“, die man beansprucht, wenn man seine innere Kraft und Orientierung verloren hat (die man konsultiert wie einen Arzt). Ist der Akku voll, ist die Kirche nicht relevant (S. 105). Man lehnt eine allwissende Selbstdarstellung der Kirche $a b$ (S. 111). Allerdings fasziniert das Geheimnisvolle und Mystische der katholischen Kirche, was einhergeht mit einer Affinität für historisch-religiös geprägte Unterhaltungsliteratur (z.B. „The Da Vinci Code“ - „Sakrileg“ oder „Die Päpstin“) oder für sagenumwobene Skandale im Vatikan (S. 105). Eine Internet-Strategie sollte die flexible, multioptionale Lebensführung der Modernen Performer nicht als oberflächlich oder modernistisch diskreditieren, aber durchaus Nachdenklichkeit über die Nebenwirkungen eines solchen Lebens kommunizieren (S. 94). Man könnte signalisieren, dass moderne Menschen jederzeit in der Kirche willkommen sind, kommen und gehen können, Entspannung und Ruhe finden können (Oasen-Modell) oder um intensiv etwas $z u$ erleben: Events, Anregungen, Austausch (S. 115). Kirchliche Riten und biblische Gleichnisse sollten in einen aktuellen Bedeutungshorizont gestellt werden - etwa in Verbindung mit Wissen aus Psychologie und Pädagogik (S. 119). Ähnlich sind die Empfehlungen im Umgang mit den Experimentalisten (S. 286): Die Kirche könnte stärker als kulturell und theologisch vielfältig kommuniziert werden. Das Geheimnis Gott/Jesus Christus ist nicht vollständig bekannt, sondern muss immer wieder neu entdeckt werden.

\section{Fazit und Empfehlungen}

Zu den bereits in Kapitel 2 aus dem Milieuhandbuch abgeleiteten Empfehlungen könnèn zusammenfassend und in der nötigen Kürze folgende Ratschläge gegeben werden:

- Im Kampf um Aufmerksamkeit im Internet waren bei der Besetzung 
wichtiger Domains (z.B. glaube.de, kirche.de, kloster.de, kirchensteuer.de) andere Kirchen, ökumenische Netzwerke oder kirchenkritische Privatpersonen schneller als die katholische Kirche. Bei aufkommenden Themen sollte man künftig wachsam sein und Domains frühzeitig besetzen. Aber nicht nur die Domains, sondern vor allem die Suchmaschinen spielen beim Besuch neuer Seiten eine wichtige Rolle. Bei zentralen Glaubensbegriffen sind katholische Kirchen-Websites kaum bei den ersten Nennungen in den Ergebnislisten dabei. Das Suchmaschinen-Marketing könnte demnach intensiver betrieben werden. Andererseits kann die katholische Kirche bei Großevents schnell einen Aufmerksamkeitssog auf zentrale Websites erzeugen - weil dafür offenbar inner- und außerhalb des Internets intensiv geworben wird und weil die Events zigtausendfach Begeisterung vor allem bei jungen Menschen hervorrufen: Beim Weltjugendtag 2005 in Köln war der Internetauftritt nach Aussage des Pressesprechers Matthias Kopp das „zentrale Kommunikationsmedium in die Welt". Alleine in den ersten 23 Augusttagen wurden 69 Millionen Zugriffe registriert. 19

- Internet-Aktivitäten, welche den kirchlichen Mainstream anpeilen, gehen am Internet-Publikum weitgehend vorbei. Vielmehr sollten Wünsche, Erwartungen und Themen kommuniziert werden, welche in modernen Milieus der Mittel- und Oberschicht anzutreffen sind. Im Wesentlichen müssen Berührungspunkte im Alltag moderner, multioptionaler Lebensstile gefunden werden.

- Kirchen-Websites sollten neben zentralen Glaubensthemen aktuelle und gesellschaftspolitische Themen aufgreifen und engagiert Stellung beziehen. Dies betrifft Themen der Medienagenda (z.B. die Debatte um den Film „Sakrileg“, welche die Website katholisch.de im Mai 2005 vorbildlich aufgegriffen hat), aber auch langfristige Themen wie Gerechtigkeit, Ökologie oder Eine Welt. Andererseits könnten individuelle Angebote an suchende Menschen kommuniziert werden - neben Großevents auch Oasen-Angebote wie Urlaub im Kloster. Zudem bietet die Verbindung Glaube/Wissenschaft Themenmöglichkeiten (z.B. zur Psychologie: „Warum glaubt der Mensch?“ - oder zur Pädagogik: „Warum brauchen Kinder in der Erziehung religiöse Orientierung?").

- Das Suchmaschinenmarketing muss darauf achten, die Aufmerksamkeit bei Glaubensthemen und bei glaubensnahen gesellschaftspoliti-

19 Vgl. Matthias Kopp: Spagat gelingender Kommunikation. Ein Erfahrungsbericht zur Kommunikationsarbeit des XX. Weltjugendtags 2005. In: Communicatio Socialis, 39. Jg. 2006, H. 1, S. 60-66, hier S. 60. 
schen Themen auf Kirchen-Websites zu lenken. Suchmaschinen bewerten Angebote besser, wenn viele Websites darauf verlinken. Deshalb sollten die vielen unterschiedlichen Websites von Diözesen, Gemeinden und Verbänden motiviert werden, intensiver untereinander zu verlinken.

- Kirchliche Events sollen multimedial im Internet begleitet werden. Insbesondere ein kluger Video-Einsatz ist unverzichtbar.

- Neue partizipative Formen sollten breit eingesetzt werden. Repräsentanten der katholischen Kirche, insbesondere Vorbilder im Glauben - zum Beispiel Ordensleute ${ }^{20}$, sollten ermutigt werden, Weblogs oder Podcasts regelmäßig zu pflegen und dafür witzige und einprägsame Namen zu finden (vgl. den „Godcast“ des Kirchenfunks auf hr3.de, Hessischer Rundfunk). Neben persönlichen Alltagsberichten und Stellungnahmen zu aktuellen Themen sollten die Blogs die Interessen und Wünsche der Internet-Nutzer aufgreifen - also weniger ein Lamento über Werte- und Moralverfall anstimmen als vielmehr positive Aspekte des Gesellschaftswandels entdecken. Ordensleute in Entwicklungsprojekten könnten in Blogs regelmäßig über Ihre Arbeit berichten. Das Image der katholischen Kirche kann unter Internet-Nutzern aufgewertet werden, wenn die persönlichen Glaubensdokumente nicht als dogmatisch, sondern als suchend empfunden werden ("Wir sind alle auf dem Weg"). Auch Jugendgruppen in Gemeinden und Verbänden könnten zur Pflege von solchen Weblogs und Podcasts angeregt werden. Insgesamt kann so ein kulturell und theologisch vielfältiges Bild der katholischen Kirche im Internet entstehen.

- Pfarrer, Gemeinde- und Verbandsvertreter sollten ermutigt - und geschult - werden, für Pfarrbriefe, Einladungen und Terminhinweise das Internet zu nutzen (nicht nur das Web, auch E-Mail und SMS). Daneben können Service-Ratschläge und Tipps für in Gemeinden engagierte Katholiken zum Download angeboten werden - von Tipps für junge Eltern (vgl. www.elternbriefe.de) bis hin zu neueren Tools des e-learnings (vgl. z.B. www.pfarrbriefservice.de).

- Und schließlich kann das Internet für ein permanentes Monitoring von Glaubens- und Kirchenthemen genutzt werden - auch unter dem

20 Gerade in Ordensgemeinschaften und Klöstern vermuten moderne Milieus eine interessante Innovationsbereitschaft (Milieuhandbuch, S. 14). Man empfindet Sympathie für Mensehen, die ihre persönliche Passion und Lebensform gefunden haben, und möchte mit ihnen ins Gespräch kommen - man wird aber schnell skeptisch, wenn man auf Ansprïche trifft, die Welt und jeden Gesprächspartner missionieren zu wollen. 
Aspekt der Krisenkommunikation. Eine einfache Möglichkeit, Themen automatisch zu verfolgen, bieten Suchmaschinen: Man abonniert zum Beispiel unter news.google.de den kostenlosen News-AlertsDienst für ein bestimmtes Stichwort und erhält dann immer automatisch eine E-Mail, wenn das Stichwort in einer von hunderten Nachrichten-Websites auftaucht.

Diese Ratschläge beruhen auf einer gegenwärtigen Analyse des Internets und der Internet-Nutzung. Da sich das neue Medium noch immer sehr dynamisch entwickelt, ist eine Revidierung auf Basis neuer Studien in einigen Jahren zu empfehlen. So wächst die Internet-Nutzung zurzeit am stärksten bei den Menschen ab 60 Jahren. ${ }^{21}$ Es ist nur eine Frage der Zeit, bis zumindest kleine Teile des kirchlichen Mainstreams durch das Internet erreicht werden können - vermutlich jedoch weniger das erzkonservative Milieu als vielmehr Gläubige, die modernen Entwicklungen gegenüber zwar skeptisch, aber aufgeschlossen sind und zum Beispiel über Kinder und Enkel den Gebrauch des neuen Mediums erfahren und lernen - und dann auch mal gerne einen E-Mail-Newsletter vom Pfarrer abonnieren oder seinen Weblog verfolgen.

21 Die Verbreitung bei den über 60-Jährigen ist mit 18 Prozent aber noch vergleichsweise gering (vgl. van Eimeren/Frees: Nach dem Boom, a. a. O., S. 365). 